\title{
Tuberous Sclerosis (tsc2+/-) Model Eker Rats Reveals Extensive Neuronal Loss with Microglial Invasion and Vascular Remodeling Related to Brain Neoplasia
}

\author{
Viera Kútna ${ }^{1} \cdot$ Libor Uttl $^{1} \cdot$ Robert Waltereit $^{2} \cdot$ Zdenka Krištofiková $^{1} \cdot$ Daniel Kaping $^{1} \cdot$ Tomáš Petrásek $^{1}$. \\ Cyril Hoschl ${ }^{1,3} \cdot$ Saak V. Ovsepian ${ }^{1,3}$
}

Published online: 9 December 2019

(C) The American Society for Experimental NeuroTherapeutics, Inc. 2019

\begin{abstract}
Tuberous sclerosis complex (TSC) is a genetic disorder characterized by frequent noncancerous neoplasia in the brain, which can induce a range of severe neuropsychiatric symptoms in humans, resulting from out of control tissue growth. The causative spontaneous loss-of-function mutations have been also identified in rats. Herein, we studied histopathological and molecular changes in brain lesions of the Eker rat model carrying germline mutation of the $t s c 2$ gene, predisposed to multiple neoplasias. Predominant subcortical tumors were analyzed, along with a rare form occurring within the pyriform lobe. The uniform composition of lesions supports the histochemical parity of malformations, with immunofluorescence data supporting their neuroglial origin. Massive depletion of mature neurons and axonal loss were evident within lesions, with occasional necrotic foci implying advanced stage of pathology. Enrichment of mesenchymal-derived cell markers with hallmarks of neurogenesis and active microglia imply enhanced cell proliferation, with local immune response. The depletion of capillaries within the core was complemented by the formation of dense mesh of nascent vessels at the interface of neoplasia with healthy tissue, implying largescale vascular remodeling. Taken as a whole, these findings present several novel features of brain tumors in Eker rat model, rendering it suitable for studies of the pathobiology and progression of primary brain tumors, with therapeutic interventions.
\end{abstract}

Key Words Neuro-oncology $\cdot$ Hamartomas $\cdot$ Glioma $\cdot$ Microglial activation $\cdot$ Nascent capillaries $\cdot$ Eker rat $\cdot$ Tuberous sclerosis complex

Electronic supplementary material The online version of this article (https://doi.org/10.1007/s13311-019-00812-6) contains supplementary material, which is available to authorized users.

Saak V. Ovsepian

saak.ovsepian@nudz.cz

Viera Kútna

viera.kutna@nudz.cz

1 Department of Experimental Neurobiology, National Institute of Mental Health, Topolová 748, 25067 Klecany, Czech Republic

2 University Hospital Carl Gustav Carus, Technical University, Department of Child and Adolescent Psychiatry, Dresden, Fetscherstraße 74, 01307 Dresden, Germany

3 Department of Psychiatry and Medical Psychology, Third Faculty of Medicine, Charles University, Ruská 87, 10000 Prague 10, Czech Republic

\section{Introduction}

Tuberous sclerosis complex (TSC) is a multisystem rare genetic disease that causes the growth of tumors in various organs. The primary morbidity is mainly due to tumors in the brain, which result in a range of neuropsychiatric signs such as drug-resistant seizures, intellectual disability, and behavioral alterations. The disorder affects over 1 million individuals worldwide, with a prevalence of 1 in 6000 newborns, with both sexes affected equally $[1,2]$. It is an autosomal dominant pathology caused by a loss-of-function mutation in tumor suppressor TSC1 or TSC2 gene located on chromosome 9q34 [3, 4] and chromosome 16p13.3 [5]. Products of TSC1 or TSC2 gene, hamartin and tuberin proteins play a key role in governing cell proliferation and differentiation, through inhibition of the mammalian target of rapamycin (mTOR) pathway [6]. Deletions and missense mutations are more frequent 
in TSC2 locus than in TSC1, with the majority of mutations in TSC1 characterized as small truncations [7, 8].

Histopathological and clinical manifestations of TSC include multiple noninvasive neoplasms, known as hamartomas (HT) in several organs, including the brain (cortical tubers, subependymal nodules, subependymal giant cell astrocytomas), kidney (angiomyolipomas), skin (angiofibromas), heart (rhabdomyomas), eye (retinal hamartomas), and lungs (lymphangioleiomyomatosis) [9, 10], with rare incidence of malignancy [11]. TSC patients often suffer neurological dysfunctions such as epilepsy, autism spectrum disorder, mental disability, motor deficits, and a range of other behavioral derangements $[2,9,12,13]$. In the cerebral cortex of TSC patients, HT is typically manifested as cortical tubers, clusters of heterotopic neurons in the white matter and giant cell astrocytomas [9]. Subependymal HTs, on the other hand, are nodular cell clusters, typically localized in the vicinity of the walls of lateral ventricles [14], with most of them remaining asymptomatic [15]. The prognosis depends on the location and severity of symptoms, which can vary on a case-by-case basis to a great degree.

Autosomal dominant mutations in $t s c 1$ or $t s c 2$ gene have been identified to induce a TSC-like phenotype in rats. Described for the first time in the context of hereditary renal adenomas and adenocarcinomas, the affected strain carries the name of a Norwegian pathologist R. Eker who first documented the conditions [16]. Although only mild neurological signs have been observed in Eker rats [17], they have been used as an animal model for human TSC, with spontaneous germline mutation of the $t s c 2$ gene. Like in humans, Eker rats developed brain lesions, making the model of considerable interest for studies of a primary brain tumor [2]. Three types of focal lesions have been documented in rats, which include subependymal hamartomas, subcortical hamartomas, and early changes related to localized disruptions of cellular architecture [2]. Subsequently, 2 additional types of lesion, i.e., cortical tubers and anaplastic gangliomas, have been documented [11]. Analysis of the cytoarchitecture and cellular content demonstrated a large number of GFAP immune-reactive cells and axons traversing the neoplastic tissue, immune-positive for SMI-31 (phosphorylated neurofilament), $\beta$-III tubulin (a marker of neurons), and myelin basic protein [2].

Although there is a general agreement on the key role of giant astrocytes in these malformations, little is known about the fate of neurons and other glial cells, as well as local and long-range axons within the neoplasia. It is not completely clear also if cell proliferation is astrocyte-selective, or involves also other cell types. Finally, no data is available on local immune response and vascular remodeling related to neoplastic changes. In this study, we show several previously unrecognized features of hamartomas in rats, verifying the utility of this primary tumor model for a range of basic and translational research, with potential therapeutic intervention.

\section{Material and Methods}

\section{Animals}

In total, 46 animals of both sexes with Long-Evans background were used. These included 12 animals aged 1 month (8 Eker $t s c 2+/-, 4$ controls - tsc $2+/+), 17$ nine-month-old rats (11 tsc $2+/-, 6$ controls) and 17 eighteen-month-old rats (11 $t s c 2+/-, 6$ controls), with 2 additional animals, which died prematurely. All rats used in our experiments were bred through crossing of $t s c 2+/-$ males (obtained from Technische Universität Dresden, Germany) with wild-type Long-Evans females. For genotyping, a tissue sample was obtained from the tail tip of every animal on postnatal days 3 or 4 . The genotypes were determined using PCR, following the protocol by Rennebeck [18]. All animals were housed in a Bio-resources facility of the Department of Experimental Neurobiology at the National Institute of Mental Health, Czech Republic, in airconditioned animal units, with 12-h light/12-h dark cycle maintained, food and water provided ad libitum. Experimental procedures involving animals have been reviewed and approved by the local Committee for Animal Protection and were conducted in accordance with the Animal Protection Code of the Czech Republic, and directive of the European Community Council on the use of laboratory animals (2010/63/EC).

\section{Histology}

Animals were deeply anesthetized with an intramuscular injection of xylazine $(30 \mathrm{mg} / \mathrm{kg})$ and ketamine $(200 \mathrm{mg} / \mathrm{kg})$ and transcardially perfused with $0.9 \%$ saline followed by $4 \%$ paraformaldehyde (Sigma-Aldrich, catalog\# P6418) in 0.1 M phosphate buffer, at pH 7.4. Brains were removed, post-fixed overnight in $4 \%$ paraformaldehyde and cryoprotected in $30 \%$ sucrose. Frozen brains were serially sectioned on a cryostat (Leica CM1860 UV) into $50 \mu \mathrm{m}$ coronal sections (1 in 5 series), which were stored in a cryoprotective solution (ethylene glycol, glycerol, and disodium phosphate in distilled water) at $-20^{\circ} \mathrm{C}$ until processed. For immunohistochemistry and histology, all brain sections were mounted on gelatin-coated slides. We used toluidine blue (Nissl; Sigma-Aldrich, USA) and hematoxylin and eosin (H\&E; Sigma-Aldrich, USA) staining to visualize neoplasia-related histopathological changes in the brain, using light microscopy (Zeiss Axio Imager Z1 microscope). For morphological analysis and characterization, we examined brain slices corresponding to tumor cross-section and related to the anatomical atlas of the rat brain [19].

\section{Immunohistochemistry and Confocal Imaging}

Free-floating brain sections were incubated with primary antibodies diluted in $0.1 \mathrm{M}$ PBS containing $0.3 \%$ Triton-X 100 overnight at $4{ }^{\circ} \mathrm{C}$. The following primary antibodies were used 
in this study: anti-Glial fibrillary acidic protein (GFAP, rabbit polyclonal, 1:5000; Dako, catalog\# Z033429), anti-Neuronal nuclear antigen (NeuN, mouse monoclonal, 1:1000, Millipore, catalog\# MAB377), anti-Doublecortin (DCX, guinea pig polyclonal, 1:3000, Millipore, catalog\# AB2253), anti-Parvalbumin (PV, mouse monoclonal, 1:3000, SigmaAldrich, catalog\# P3088), anti-Tyrosine hydroxylase (TH, rabbit polyclonal, 1:2000, Millipore, catalog\# AB152), antiIba1 (rabbit polyclonal, 1:3000, Wako, catalog\# 019-19741), anti-Nestin (mouse monoclonal, 1:200, Millipore, catalog\# MAB353), anti-Vimentin (chicken polyclonal, 1:1000, Millipore, catalog\# AB5733), and anti-Endothelial cell antibody (RECA-1, mouse monoclonal, 1:400, Abcam, catalog\# ab9774). Before incubation with anti-Nestin antibody (rabbit monoclonal, 1:100, Abcam, catalog\# ab221660), we performed heat mediated antigen retrieval by using citrate buffer (pH 6.0) at $95{ }^{\circ} \mathrm{C}\left( \pm 3{ }^{\circ} \mathrm{C}\right)$ for $15 \mathrm{~min}$ and blocking of nonspecific protein activity with $10 \%$ normal donkey serum (Millipore, catalog\# S30) and 0.3\% Triton-X 100 for $30 \mathrm{~min}$ at room temperature. After incubation with primary antibodies, sections were extensively rinsed in $0.1 \mathrm{M}$ PBS and incubated with secondary antibodies in $0.1 \mathrm{M}$ PBS containing $0.3 \%$ Triton-X 100 for $2 \mathrm{~h}$ at room temperature. The following secondary antibodies conjugated with fluorophores were used, according to the chosen primary antibodies (Donkey anti-guinea pig AF488 - catalog\# 706-545-148, Donkey antirabbit AF488 - catalog\# 711-545-152, Donkey anti-mouse AF594 - catalog\# 715-585-151, Donkey anti-rabbit AF594 catalog\# 711-585-152, Donkey anti-chicken AF488 - catalog\# 703-545-155, 1:500, Jackson ImmunoResearch). After incubation with secondary antibodies, sections were rinsed in $0.1 \mathrm{M}$ PBS and coverslipped in ProLong Gold Antifade Reagent with DAPI (Cell Signaling Technology, catalog\# 8961). Fixed immunofluorescence samples were viewed with a Zeiss Axio Imager Z1 fluorescent microscope (Carl Zeiss, Jena, Germany) or Leica TCS SP8X confocal system (Leica Microsystems Mannheim, Germany). The images were acquired using appropriate excitation lines and filters, which were analyzed in AxioVision Rel 4.8 software (Carl Zeiss), LAS AF software (Leica Microsystems), and ImageJ 1.47 software (NIH). The brightness and contrast of images have been adjusted in a standardized manner for all of the images as described [20]. All images and graphs were generated and assembled in figures using Igor Pro (6.37 WaveMetrics Inc) and Adobe Illustrator (Adobe Systems, San Jose, CA).

\section{Data Analysis and Statistical Significance}

Histochemical data were obtained from 46 rats in total, whereas for immunofluorescence, 5 of 18-month-old rats were used. In all experiments, the anatomically matching contralateral side of the brain has been used as control. For intensity analysis and cell counting, multiple regions of interest from multiple confocal sections have been used. Raw RGB images were converted into 8 bit black and white format with Image $\mathrm{J}$ and used for quantification. For cell counting, images were thresholded using an in-built macro with cells counted in Image $J$ as described in our previous study [21]. Cell type-specific fluorescence labeling was used for measuring the signal intensity and counting various cell types, whereas DAPI labeling was applied for counting the total cell number. Signal intensity analysis was carried out from multiple randomly defined regions of interest, from 12 to 16 optical sections of multiple animals, using signal intensity macro of ImageJ, with mean intensity values tabulated and plotted subsequently in graphs. For analysis of fluorescence areas, data were acquired using the area measurement function of ImageJ. Co-localization analysis was carried out on grayscale images, using the same regions of interest from green and blue channels, with co-localization index calculated and graph generated with ImageJ as described [22]. For analysis of microglial branching, we applied Sholl analysis as specified in our previous report [20]. All measurements are presented as individual data points, with means \pm SEM defined where appropriate. Unpaired and paired Student's $t$ tests were applied for comparison of different groups using Igor Pro, with statistical significance assessed using paired or unpaired Student's $t$ test. $P<0.05$ has been defined as a statistically significant difference.

\section{Results}

\section{Distribution and Gross Examination of Brain Neoplasia in Eker Rats}

We have analyzed in total 17 rats of 18 months of age, of which 11 had $t s c 2+/-$ and $6 t s c 2+/+$ genotypes. Two $t s c 2+/$ - animals died at the age of 15 months, in which necropsy showed hypothalamic tumors. Neither $t s c 2+/-$ nor $t s c 2+/+$ rats included in this analysis displayed overt behavioral abnormalities or seizures. Careful examination of control brains of age-matched rats showed no neoplasia or any signs of pathology in cortical and subcortical structures. We also could not find lesions in younger rats ( 1 and 9 months old, not shown), in agreement with previous reports. In the remaining 11 old Eker rats, we observed neoplastic changes in 5 animals, of which 4 were males and 1 female. In 4 animals, we found subcortical tumors in the cerebrum, whereas in 1 rat, the tissue malformation was located in the olfactory area of pyriform lobe. Figure 1 (a1-e4) illustrates representative cross-sections of toluidine blue-stained hamartomas in different brain locations. As can be seen, neoplasms in the anterior olfactory area were located next to the commissure, invading into dorsal tenia tecta (Fig. 1a); at the border of dorsal peduncular cortex a ventral and medial orbital cortex (Fig. 1b); beneath fimbria in the dorsal striatum (Fig. 1c); in cingulum coalesced with 

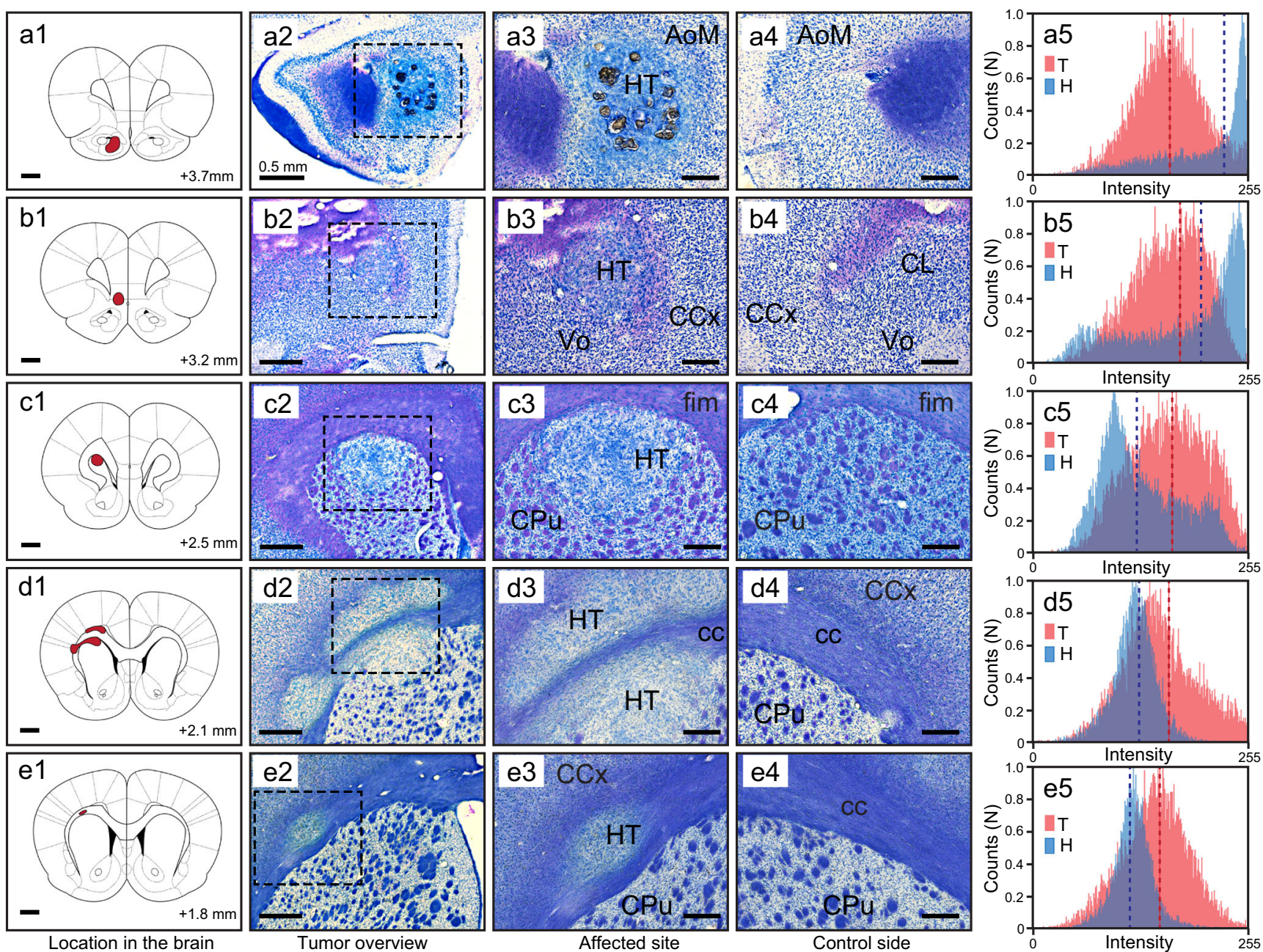

Fig. 1 Representative microphotographs of toluidine blue-stained neoplasia in different brain locations. Schematic representations of brain coronal sections containing lesions (in red) in different compartments (a1-e1). Low power micrographs presenting an overview of lesions (a2-e2) with zoomed-in images (a3-e3) and anatomically matching intact (control) side (a4-e4). Note that brain lesions in the anterior olfactory area contain multiple calcifications $(\mathrm{a} 2, \mathrm{a} 3)$. Neoplasms in the ventral orbital cortex (b2, b3), in the dorsal striatum (c2, c3), in cingulum and corpus callosum $(\mathrm{d} 2, \mathrm{~d} 3)$ and in corpus callosum - external capsule (e2, e3) Graphical representation of signal distribution in tumor areas ( $\mathrm{T}$, red) and matching normal healthy tissue ( $\mathrm{H}$, blue) (a5-e5). Of note, signal distribution from HT showed strong cross-sample consistency in all locations, unlike signal distribution of control healthy tissue. AoM, anterior olfactory area medial part; fim, fimbria; cc, corpus callosum; $\mathrm{CCx}$, cortex; $\mathrm{CPu}$, caudate putamen; HT, hamartoma; Vo, ventral orbital cortex. Scale bars: $1 \mathrm{~mm}$ (a1-e1), $500 \mu \mathrm{m}$ (a2-e2), $100 \mu \mathrm{m}$ (a3-e3, a4-e4) external capsule (Fig. 1d) and within the corpus callosumexternal capsule (Fig. 1e). Closer inspection of lesions releveled disorderly arranged cells with diffuse boundaries of neoplasm, with a large number of cells possessing pleomorphic small nuclei. Signs of apoptosis were also regularly observed within neoplastic tissue mass. Semi-quantitative analysis and comparison of signal distribution in lesions and in anatomically matching healthy tissue showed strong crossconsistency between different lesions, independent of their location, with remarkably constant intensity distribution graphs, unlike signal distribution graphs of intact control side, which showed location-specific variability (Fig. 1 a1-5, e1-5) (Table 1).

\section{Astrogliosis, Neuronal Loss, and Axonal Degeneration}

To investigate more closely the cellular composition of neoplasms with related histopathological changes, we carried out triple immunostaining with microscopic analysis. Figure $2 \mathrm{a}-\mathrm{c}$ shows typical immunofluorescence micrographs with neuron and astrocyte-specific markers, NeuN and GFAP, respectively, combined with nuclear DAPI staining of 2 typical tumors. At low magnification, a strong increase in GFAP signal is evident within lesions accompanied by depletion of neurons (Fig. 2 a, b). On high power images, densely intermingled large GFAPpositive astrocytes are visible, with diffuse and gradual transition at the borderline with surrounding intact parenchyma 
Table 1 Summary of the signal intensity distribution in neoplastic tissue and in anatomically matching control regions

\begin{tabular}{llllll}
\hline & \multicolumn{2}{l}{ Hamartoma } & & & \multicolumn{2}{c}{ Control } \\
\cline { 2 - 3 } \cline { 5 - 6 } & Mean & SD & & Mean & SD \\
\hline Case 1 & 155.9 & 44.8 & & 225.1 & 53.1 \\
Case 2 & 165.7 & 41.3 & & 192.7 & 67.3 \\
Case 3 & 165.8 & 41.1 & & 128.3 & 39.9 \\
Case 4 & 179.9 & 46.0 & & 127.9 & 31.7 \\
Case 5 & 148.3 & 32.0 & 113.8 & 16.9 \\
\hline
\end{tabular}

marked by changes in the relative ratio of NeuN and GFAP markers (Figure 2 a1-2; b1-2, c). The significant increase in GFAP expression with overall changes in cell numbers in neoplastic tissue is clearly visible in summary histograms, in which mean intensities of NeuN and GFAP signals are plotted along with the results of cell counting analysis. Remarkably, despite changes in GFAP and NeuN signals, the overall intensity of DAPI signal over multiple analyzed regions of interests remained unaltered (Fig. 2d). Due to strong GFAP signal, counting of astrocytes within lesions was not feasible, whereas the total number of neurons was greatly reduced. Interestingly, even though the intensity of DAPI remained consistent between neoplastic and control tissue, nuclear counting using DAPI signal showed a significant increase in the number of cells, with numerous pleomorphic small nuclei visible within lesions (Fig. 2e).
The occurrence of seizures in TSC-affected patients could partly result from disruption of the inhibitory circuits, and alterations of the fine balance between excitatory and inhibitory synaptic drives [23]. They also may result from tumorrelated remodeling of long-range neuromodulator projections, from the brain stem nuclei [24]. Immunofluorescence staining of neoplasia in Eker rats with parvalbumin-specific marker showed significant loss of GABAergic neurons expressing this calcium buffering protein (Suppl. Fig. 1 a, b). Likewise, extensive loss of tyrosine hydroxylase (TH)-positive longrange catecholaminergic axons was evident in neoplasia. Figure 3(a-f) shows representative triple stained micrographs from $t s c 2+/-$ lesion and anatomically corresponding regions of $t s c 2+/+$ control rats, demonstrating fragmentation and loss of catecholaminergic axons within the neoplastic tissue, together with strong depletion of neurons. These changes are clearly reflected in intensity and area distribution histograms, showing a strong reduction in NeuN and $\mathrm{TH}$ signals, despite that the DAPI signal remained unaffected (Fig. 3c, d).

\section{Activation of Neuro-glial Progenitor Cells}

Expression of mesenchymal-derived cell markers with hallmarks of neurogenesis and metastatic activity is amongst the most recognized feature of human gliomas [25]. Described above increase in the number of DAPI-positive cells along with neuronal loss implies enhanced proliferation of glial cells within lesions. To investigate if neoplasia in $t s c 2+/-$ rats share these hallmarks
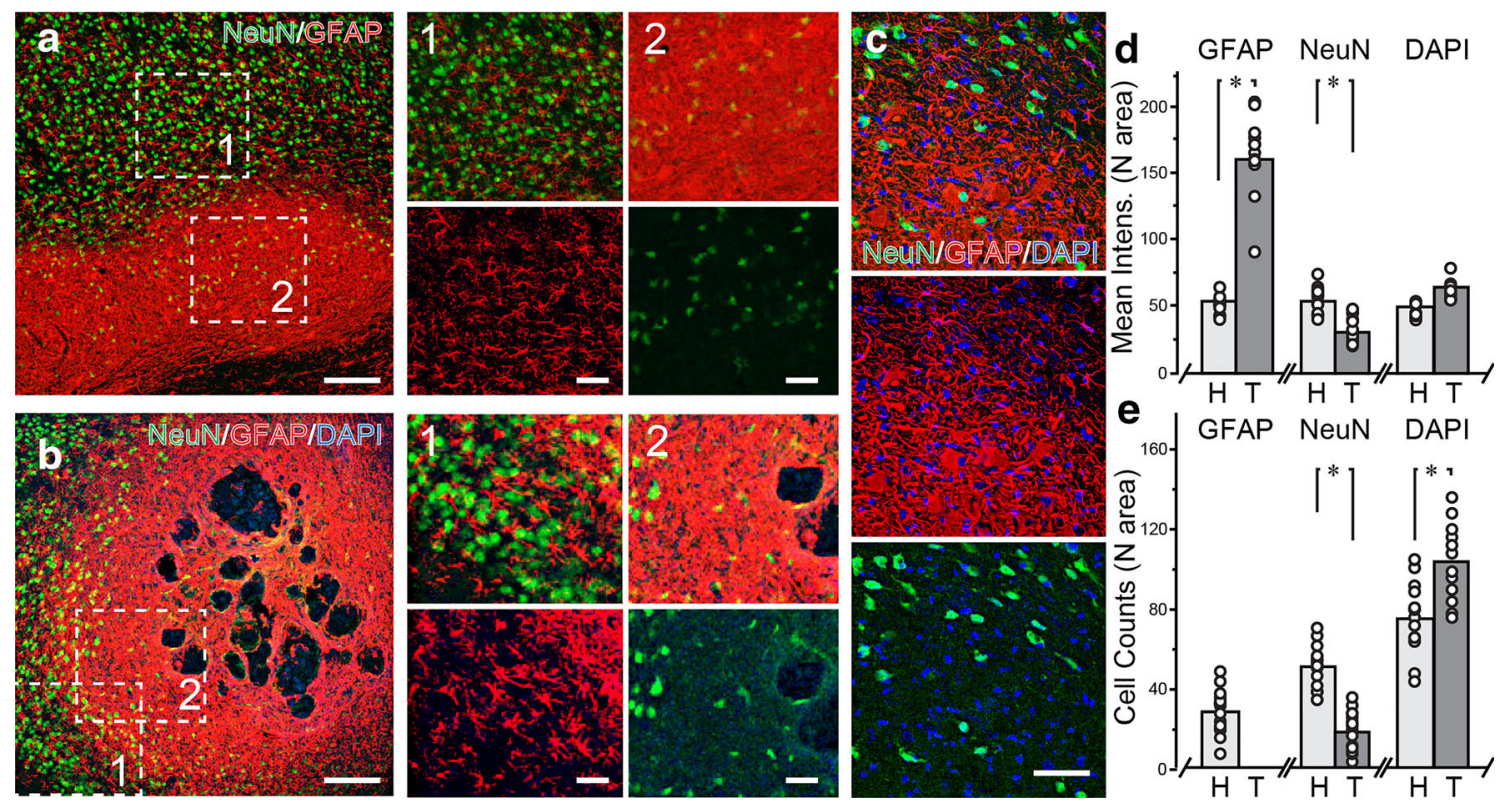

Fig. 2 Immunofluorescence visualization of NeuN- and GFAP-positive cells in hamartomas. High GFAP and weak NeuN expression in 2 typical hamartomas in the ventral motor and external capsule region (a), and in the anterior olfactory area (b). Scale bar $100 \mu \mathrm{m}$. At higher magnification, robust GFAP immunoreactivity inside lesion area is evident, with differences in density of NeuN-positive neurons also clearly visible; scale bar
$25 \mu \mathrm{m}(\mathbf{c})$. Mean intensity analysis of multiple regions of interests shows enhanced GFAP and reduced NeuN signals compared with healthy tissue (d). The number of GFAP-positive astrocytes in hamartoma could not be counted due to the high signal intensity, but the number of NeuN-positive cells is reduced (e). (H, healthy tissue; T, tumor). $* p<0.05$ 
a

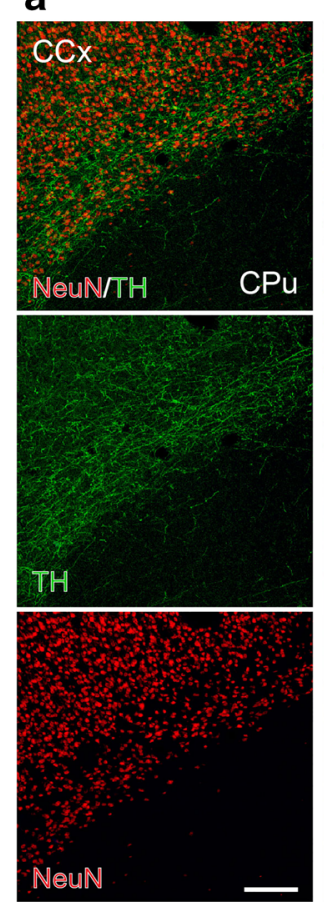

b
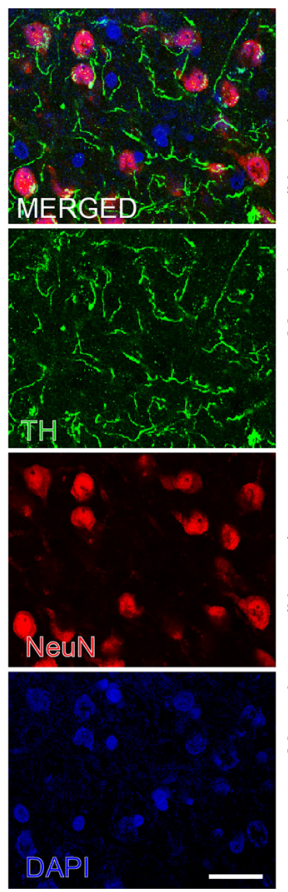

Fig. 3 Visualization and analysis of the effects of hamartomas on longrange TH-positive axons. Representative triple immunofluorescence micrographs showing the density and distribution of TH-positive axons in intact $(\mathbf{a}, \mathbf{b})$ and neoplasia area $(\mathbf{e}, \mathbf{f}) . \mathrm{CCx}$, the cerebral cortex. $\mathrm{CPu}$, caudate putamen. Low $(\mathbf{a}, \mathbf{e})$ and high power $(\mathbf{b}, \mathbf{f})$ micrographs. Note a dramatic reduction in the density of structural elements labeled positive

of gliomas, and to verify the progenitor cells of neoplasia, immunofluorescence labeling with co-localization analysis has been carried out using anti-vimentin and anti-nestin antibodies (Fig. 4). During neurodevelopment, high expression of nestin is attributed to neural stem/progenitor cells, which is followed by nestin degeneration and clearance after cell differentiation, marking the maturity [26]. Accordingly, in control samples, we detected highly localized expression of nestin within the subventricular zone of adult rats, known to be the primary site of the origin of adult-born olfactory granule cells (Suppl. Fig. 2 a, b). In $t s c 2+/-$ rats, a highly localized increase in nestin was evident within the neoplastic tissue, as visible in Fig. 4 (a-d). Moreover, the high expression of nestin in the lesion area was associated with strong enrichment of neoplastic tissue with vimentin, known as a marker for immature proliferating cells (Fig. 4a-c). Importantly, the intensity distribution measurements and mapping of these 2 proteins showed significantly enhanced expression of both in neoplasia, with their high co-localization coefficient (Fig. 4e, f). Closer inspection of lesions showed that both vimentin and nestin were localized primarily in cells with nondifferentiated, spindle-like or multipolar appearance, with pleomorphic small nuclei. It is noteworthy that the expression level of vimentin has been used as a marker for the low-grade gliomas, with its low levels predicting a longer survival and better responsiveness to temozolomide therapy [27]. e
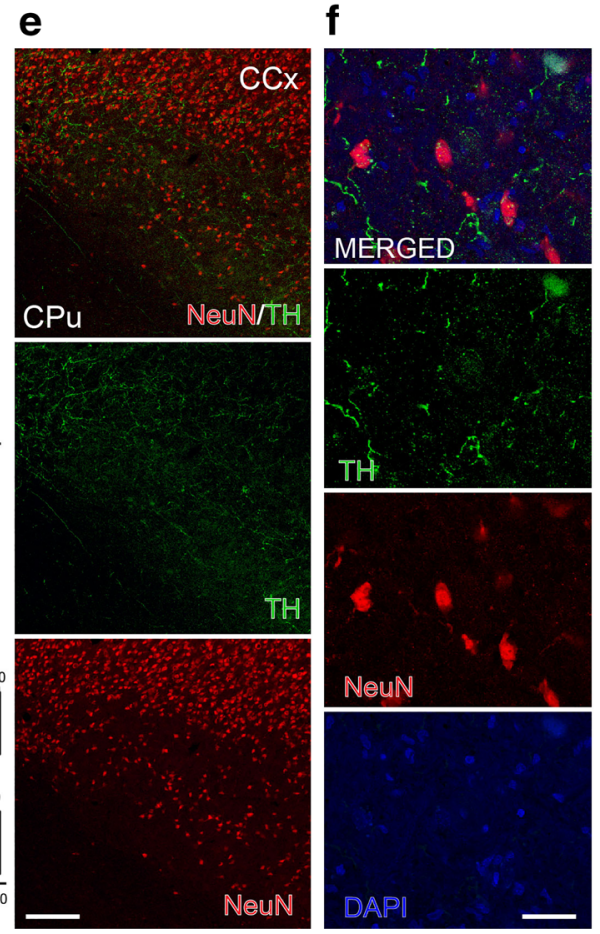

for $\mathrm{TH}$, with a strong reduction in number of NeuN-positive neurons in the tumor-affected region. Graphical representation of changes in the mean intensity of TH and NeuN signals (c) and mean intensity and area of TH fluorescence signal (d). Scale bars: A, E-100 $\mu \mathrm{m} ; \mathrm{B}, \mathrm{F}-25 \mu \mathrm{m}$. (a.u., arbitrary units; $\mathrm{CCx}$, cortex; $\mathrm{CPu}$, caudate putamen). ${ }^{*} p<0.05$

\section{Invasion of Microglia and Macrophage Activation}

To investigate the local immune response to lesions in the brain of $t s c 2+/-$ rats, we used triple stainings with allograft inflammatory factor 1, also known as ionized calciumbinding adapter molecule, or Iba1. Amongst brain cells, Iba1 is strongly and specifically expressed in microglia and in circulating macrophages [28], playing an essential role in reorganizing the cytoskeleton and plasmalemma during phagocytosis. Figure 5 presents the results of immunofluorescence labeling with semi-quantitative scoring of Iba1, NeuN, and DAPI expression in a typical lesion of Eker rat. Similar to GFAP/NeuN staining, a pronounced increase in Iba1 expression in neoplastic tissue was marked with a massive loss of NeuN labeled cells readily visible (Fig. 5a-d). Compared to the resting microglia in intact tissue, showing orderly and small cell bodies along with highly ramified branching processes, in lesion area, most of the microglial cells are transformed into active phagocytic cells, with retracted processes and "amoeboid" morphology, reminiscent to activated macrophages (Fig. 5b, c, and e). The latter is also visible from Sholl analysis graph, as well from enrichment of Iba1-positive spindle-like, intermediate polygonal and giant multipolar cells within lesions. 

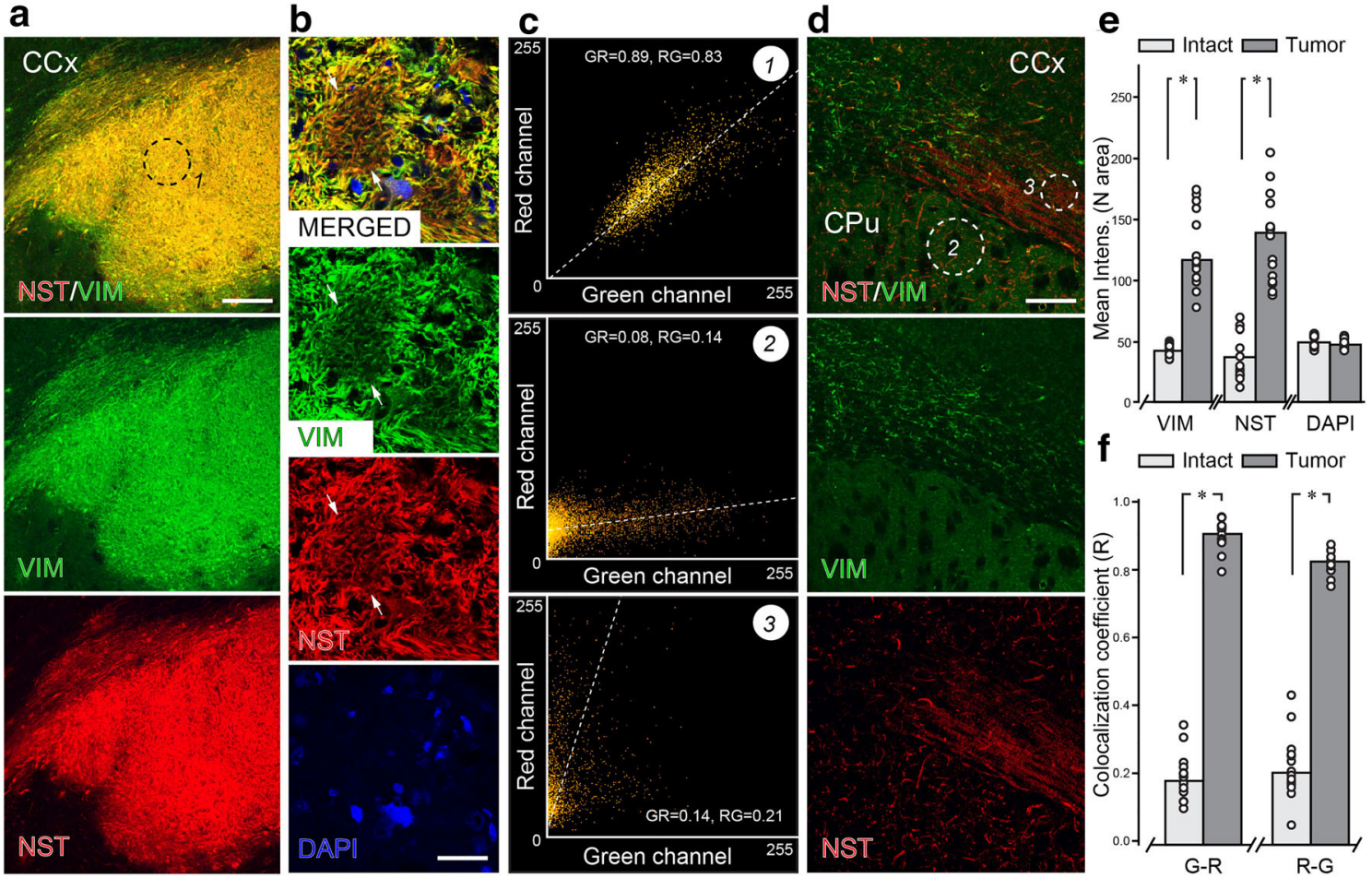

Fig. 4 Immunoreactivity of neoplastic tissue for neuron and glial progenitors and mesenchymal cell markers. Typical double and triple fluorescence micrographs showing enrichment of neoplastic tissue with mesenchymal cell and metastatic marker vimentin, and developing neuronal marker nestin, respectively $(\mathbf{a}, \mathbf{b})$ in comparison with the anatomically matching healthy side (d). $\mathrm{CCx}$, cerebral cortex. $\mathrm{CPu}$, caudate putamen. Scale bars $100 \mu \mathrm{m}(\mathbf{a}, \mathbf{d})$, and $25 \mu \mathrm{m}(\mathbf{b})$. Very high

\section{Vascular Remodeling and Angiogenesis}

Development of neoplastic tissue requires extra nutrition and oxygen supply, warranting the formation of new blood vessels, by rerouting or remodeling the existing vasculature [29]. To investigate if the out of control tissue growth in nonmetastatic brain tumors of $t s c 2+/-$ rat involves largescale vascular remodeling, we applied customary $\mathrm{H} \& \mathrm{E}$ staining as well as immunofluorescence labeling, using mature endothelial cell marker RECA-1. Suppl. figure 3 compares representative $\mathrm{H} \& \mathrm{E}$ images of healthy control tissue and tissue affected by neoplasia $(\mathrm{a}-\mathrm{c})$. Enhanced bluepurple labeling of tumor-affected region is consistent with increased DNA-RNA levels, indicative of intense tissue proliferation, with dense clusters of actively diving cells visible. Blue-purple labeling appears enhanced in putative endothelial cells of small blood vessels, in line with active proliferation associated with angiogenesis. Figure 6 shows the results of comparative analysis of typical cross-sections of lesion area and anatomically matching control tissue stained for RECA-1 and GFAP (Fig. 6 a, b). Remarkable changes general architecture and morphometric characteristics of feeding vasculature are readily visible, with the core of the lesion showing dramatic depletion of RECA-1- degree of co-localization of vimentin and nestin signals in neoplasia is in agreement with their co-expression in the same populations of cells $(\mathbf{c}, \mathbf{f})$. Note that in the healthy tissue of adult brain, the expression of these proteins is very weak and reveals a distinct distribution (d). Intensity distribution analysis histogram showing significantly enhanced expression of both proteins of interest in lesion area $(\mathrm{E}) . * p<0.05$

positive microvasculature, especially prominent in the inner compartments of the tumor. The latter is likely to contribute towards occasional necrotic foci within neoplasia (e.g., Fig. 1a). The dissolution of vessels in the tumor core is associated with notable increase in the density of small diameter vessels encircling the growing edge, implying localized angiogenesis and formation of new vessels to support rapidly expanding compartments of neoplasia. The intensity and area distribution analysis and comparison of the core and the edge of neoplastic tissue with intact surroundings are presented in Fig. 6c, d, demonstrating changes in the mean intensity of RECA-1 staining as well as in the proportion of small and large diameter blood vessels related with the tumor mass (Fig. 6d). To determine if RECA1positive vascular endothelial cells are enriched with nestin, we carried out a triple immunofluorescence labeling for RECA-1 and nestin, and nuclear marker DAPI. The results of these experiments are summarized in Suppl. figure 4. Overall, our results suggest that although RECA-1 allows distinction of mature endothelial cells in neoplastic tissue, differentiation of actively dividing immature endothelial cells based on nestin or cell appearance is problematic, due to enrichment of the neoplastic tissue with atypical cells, including glial cells and neuro-glial progenitors. 

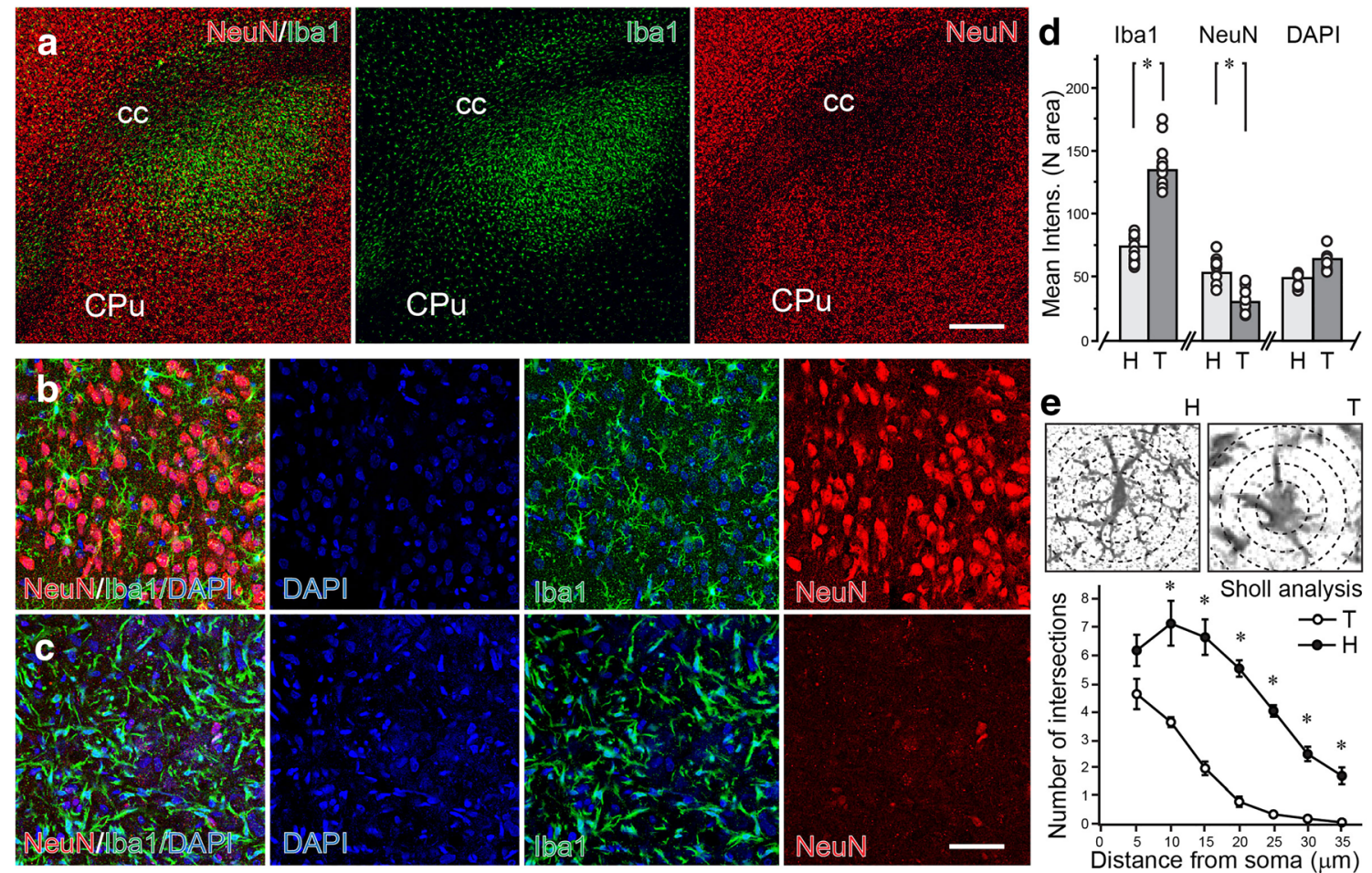

Fig. 5 Activation of microglia and accumulation of macrophages within neoplastic tissue. Representative double immunofluorescence micrographs illustrating the massive loss of NeuN-positive cells with accumulation of Iba1 reactive, putative microglia and macrophages within the lesion (a). cc, corpus callosum; $\mathrm{CPu}$, caudate putamen. Scale bar $100 \mu \mathrm{m}$. Triple immunofluorescence higher power micrographs presenting a closer view on cellular distribution and activation of Iba1positive profiles $(\mathbf{b}, \mathbf{c})$ demonstrating local proliferation of immune cells with phagocytic response within the tumor. Scale bar $25 \mu \mathrm{m}$. Summary histograms showing relative changes of neuronal and microglial markers in neoplasia (d) with results of Sholl analysis verifying the activation of microglia within the neoplastic tissue (e). $* p<0.05$

\section{Discussion}

In this study, we combined traditional histochemical approaches with immunofluorescence microscopy to elucidate cellular and molecular hallmarks related to neoplasia in the brain of Eker rats, carrying a loss-of-function mutation of the $t s c 2$ gene $(t s c 2+--)$. From 5 analyzed tumors, 4 were subcortical, located in the dorsal cerebrum, whereas 1 was found within the pyriform lobe, a location not reported previously. The cellular composition and molecular profile analysis have revealed strongly enhanced GFAP expression and strong decrease in NeuN signal, in line with a major astrocytic element in neoplasia and extensive degeneration of mature neurons. The out of control tissue growth in the brain was also associated with significant fragmentation of both, local and longrange axons, with their loss. Localized changes in neuronal and astrocyte hallmarks were associated with increased levels of mesenchymal cell markers and Ibal signal, suggesting enhanced cell proliferation and microglia invasion. With occasional necrotic lesions and strong depletion of microvasculature in the tumor core implying advanced pathology, with clear signs of vascular remodeling, and angiogenesis also evident.
In agreement with results of earlier reports [11, 17], neoplastic changes in the brain of Eker rats showed strict agedependence, being present only in old animals, with young rats showing no signs of brain pathology. Interestingly, earlyonset hamartomas have been also reported in Eker rats $(t s c 2+/$ $-)$, but only in experiments with prenatal expose to hydroquinone [30], or postnatal irradiation [31, 32]. Based on histopathological features, Yeung et al. (1997) classified hamartomas in Eker rats according to their preferential location into 2 groups: subependymal and subcortical. Mizuguchi et al. (2000) subsequently described 2 additional lesion types, cortical tubers, and anaplastic gangliomas. The most common type of brain lesions in Eker rats are subcortical hamartomas. Subependymal neoplasia was described in Eker rats of Fischer 344 background [2], although only in a single case of cortical tuber was found in 19-month-old Eker rats [11]. Subependymal giant cell astrocytomas belong to the most frequent tumors in TSC patients [33], which in their majority appear in lesions enriched with spindle-like, intermediate polygonal and giant multipolar cells [34].

Consistent with previous reports $[2,11]$, astrocytes also show a high level of activity in neoplasia in our samples. It is important to note, however, that pure spontaneous 

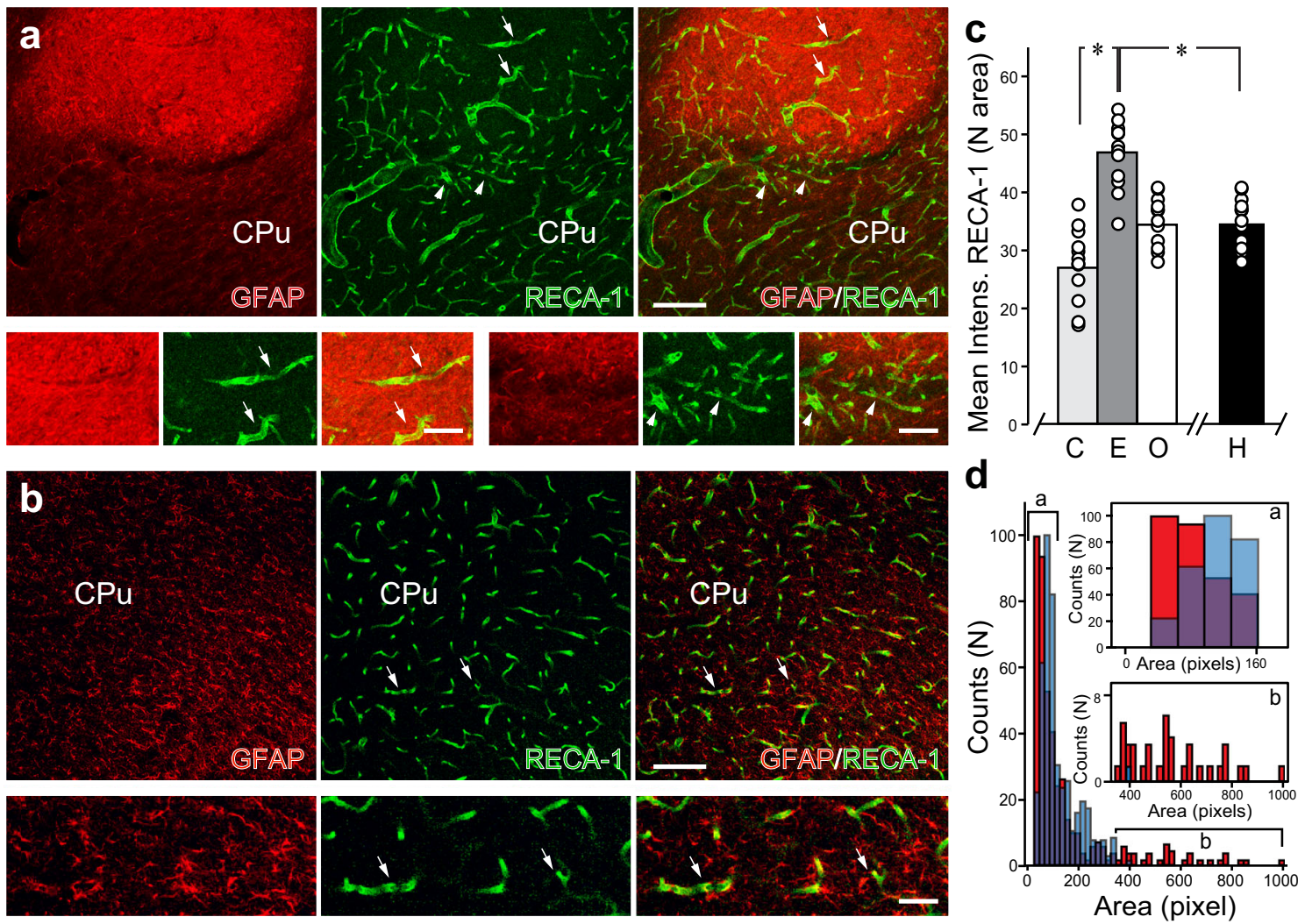

Fig. 6 Loss and remodeling of the microvasculature related to brain neoplasia. Low and high magnification GFAP and RECA-1 double immunofluorescence micrographs of a typical lesion in the rat brain, and anatomically matching intact region showing tumor-related changes in density and distribution of RECA-1 labeled vascular profiles within the lesion area and surrounding parenchyma $(\mathbf{a}, \mathbf{b})$. $\mathrm{CPu}$, caudate putamen. Scale bars $100 \mu \mathrm{m}$ and $20 \mu \mathrm{m}$. Note disproportionately larger vessels (white arrows) in the core of the lesion with loss of micro-vessels,

although the density of small vessels at the interface of the tumor and healthy tissue is notably enhanced. Graphical representation of changes in mean intensity of RECA-1 signal in the tumor core, edge and out of the lesion area $(\mathbf{C}, \mathbf{E}$, and $\mathbf{O}$ respectively) compared to the healthy tissue $(\mathbf{H})$. (d) A summary histogram of the RECA-1-positive area distribution in micrographs of intact (blue) and tumor-affected (red) brain section, showing increase in population of small and large vessels. ${ }^{*} p<0.05$

astrocytomas in rats, unlike those in humans, are uniformly immune-negative to GFAP $[35,36]$. Hence, a strong increase in GFAP signal in neoplasia in Eker rats shown herein is likely to reflect an increased level of reactive astrocytes within the tumors stroma, which comprises in addition to astrocytes other neoplastic host cells, or their precursors. Using immunofluorescence microscopy, we show that in addition to enrichment with GFAP, the growing tissue mass also expresses very high levels of nestin, a filament protein recognized as a marker of neural stem cell progenitors [37], human CNS tumors [38, 39] as well as hallmark of premature endothelial cells [40]. Nestin expression was observed in proliferating endothelial cells in vitro and in vivo [41] with high rate of growth [42], but not in mature endothelial cells [41]. High expression of nestin is also a hallmark for mesenchymal cells [43] and epithelia-tomesenchymal transition during development and metastatic process, verifying the presence of undifferentiated precursor cells in the stroma of neoplasia. Furthermore, in contrast to the data reported by Mizuguchi et al. (2000), we observed a strong enrichment of intermediate filament protein vimentin in neoplastic region of our samples. The latter is known as a marker

for radial glia expressed in undifferentiated neural stem cells, as well as in glial precursors. Of note, the expression of vimentin in the adult brain under physiological conditions is constrained to selected brain regions and maintained at low levels [44]. Its increase in neoplasia of $t s c 2+/-$ Eker rats, hence, implies not only tumor-related activation of endothelial cell proliferation but also strong tissue growth, reminiscent to those found in malignant glioblastoma and anaplastic astrocytoma [45]. It is worth noting that some compartments of neoplasia of our samples also express doublecortin (DCX), a marker for neuronal progenitors of the subventricular zone (SVZ), as well as migrating neurons. Of note, the expression of DCX was previously described only in giant cells in the cortex of TSC patients [46, 47], whereas the presence of DCX-positive cells in human hamartomas remains to be shown. Altogether, although these observations suggest that out of control growth of tumors in Eker rat could primarily originate from the progenitor of astrocytes and neuronal cells undergoing abnormal differentiation, the importance of microglia invasion and macrophages in affected tissue should be also considered. The latter is supported 
by our immunofluorescence data demonstrating strong enrichment of microglia in lesions, with all tested samples displaying distinct positivity for microglia-macrophage Iba1 marker [48].

Similar to the vast majority of solid brain tumors and in line with previously reported data, the core of neoplasia in samples analyzed in this study appears depleted of neurons, with sparse presence of neuronal markers, such as neurofilament $[2,11]$ and $\beta$ III tubulin [2], and greatly reduced numbers of NeuN-positive cells. Likewise, we observed a remarkable decrease in parvalbumin-positive neurons, which represent primarily chandelier and basket GABAergic interneurons [49]. Finally, we have observed a depletion of tyrosine hydroxylase-positive axons within the lesion, which are largely of subcortical origin. The extensive tissue growth with alterations in cellular composition and architecture evidenced by the enhanced expression of cellular proliferation hallmarks implies significant adjustments in nutrient and gas supply to the neoplasia [50]. Accordingly, we observed remarkable changes in vascularization of the tumor and surrounding tissue, with unduly large vessels and pronounced loss of capillaries clearly distinguishable in the core, whereas the edge of the neoplastic tissue was marked by a concentration of small diameter nascent vessels, as revealed with immunostaining using anti-RECA-1 antibody. Changes in vascular architecture and distribution within affected regions suggest extensive vascular remodeling, a well-recognized feature of brain neoplasia [29]. It remains to be shown, however, if changes in vascularization of brain tumors of Eker rats are associated with alterations of the permeability of micro-capillaries and disruption of the blood-brain barriers.

In summary, in this report, we have independently verified several well-established characteristics of neoplasia in $t s c 2+/-$ Eker rats, and report new features, closely replicating histopathological and molecular traits of TSC human patients. Together with functional data showing reoccurring epileptic seizures and a range of other neurological signs in humans, our data support the use of described herein model for investigating a range of neuropathological changes associated with neoplasia in the brain, and for translational studies targeting different facets of the disease. Unlike widely used acute xenografts comprised of a uniform cell mass in animal models with strongly suppressed immune response, with a very brief history of the pathology, the model described herein mimics true histopathological complexity and history of brain neoplasia in remarkable detail, with fully maintained neuro-immune response, along with local cellular and humoral changes. Through the use of advanced drug targeting and delivery systems, it is hoped that this model would be suitable for future studies of therapeutic delivery and management of uncontrolled tissue growth for tackling hamartomas and related neoplasia under basic and translational settings, as well as in neuroimaging studies.
Acknowledgments This study was supported by the project Sustainability for the National Institute of Mental Health, under grant number LO1611, with financial support from the Ministry of Education, Youth and Sports of the Czech Republic under the NPU I program. The authors thank Mrs. Jana Babáčková for excellent technical assistance.

Author Contribution Statements V.K., L.U., and T.P. designed the experiments; D.K. conceived the original idea; V.K. and L.U. contributed to sample preparation; V.K. performed the experiments; R.W. and Z.K. provided biomaterials; S.V. O. and V.K. analyzed the results; V.K., S.V. O., T.P., R.W., Z.K., and C.H. contributed to the final version of the manuscript with input from all authors; S. V, O. supervised the project.

Abbreviations TSC, tuberous sclerosis complex; mTOR, mammalian target of rapamycin; $H T$, hamartoma; $D C X$, doublecortin; $P V$, parvalbumin; $T H$, tyrosine hydroxylase; $P B S$, phosphate buffer salt; $G F A P$, glial fibrillary acidic protein; $D A P I, 4^{\prime}, 6$-diamidino-2phenylindole; $G A B A$, gamma amino butyric acid; $A o M$, anterior olfactory area medial part; fim, fimbria; $c c$, corpus callosum, $\mathrm{CCx}$, cortex; $C P u$, caudate putamen; $V o$, ventral orbital cortex; $E C$, external capsule

\section{References}

1. Osborne, J.P., A. Fryer, and D. Webb, Epidemiology of tuberous sclerosis. Ann N Y Acad Sci, 1991. 615: p. 125-7.

2. Yeung, R.S., C.D. Katsetos, and A. Klein-Szanto, Subependymal astrocytic hamartomas in the Eker rat model of tuberous sclerosis. Am J Pathol, 1997. 151(5): p. 1477-86.

3. Fryer, A.E., et al., Evidence that the gene for tuberous sclerosis is on chromosome 9. Lancet, 1987. 1(8534): p. 659-61.

4. van Slegtenhorst, M., et al., Identification of the tuberous sclerosis gene TSC1 on chromosome 9q34. Science, 1997. 277(5327): p. 8058.

5. Kandt, R.S., et al., Linkage of an important gene locus for tuberous sclerosis to a chromosome 16 marker for polycystic kidney disease. Nat Genet, 1992. 2(1): p. 37-41.

6. Tee, A.R., et al., Tuberous sclerosis complex-1 and -2 gene products function together to inhibit mammalian target of rapamycin (mTOR)-mediated downstream signaling. Proc Natl Acad Sci U S A, 2002. 99(21): p. 13571-6.

7. Jones, A.C., et al., Molecular genetic and phenotypic analysis reveals differences between TSC1 and TSC2 associated familial and sporadic tuberous sclerosis. Hum Mol Genet, 1997. 6(12): p. 215561.

8. Kozlowski, P., et al., Identification of 54 large deletions/duplications in TSC1 and TSC2 using MLPA, and genotype-phenotype correlations. Hum Genet, 2007. 121(3-4): p. 389-400.

9. Curatolo, P., R. Bombardieri, and S. Jozwiak, Tuberous sclerosis. Lancet, 2008. 372(9639): p. 657-68.

10. Davis, P.E., et al., Presentation and Diagnosis of Tuberous Sclerosis Complex in Infants. Pediatrics, 2017. 140(6).

11. Mizuguchi, M., et al., Novel cerebral lesions in the Eker rat model of tuberous sclerosis: cortical tuber and anaplastic ganglioglioma. J Neuropathol Exp Neurol, 2000. 59(3): p. 188-96.

12. Gillberg, I.C., C. Gillberg, and G. Ahlsen, Autistic behaviour and attention deficits in tuberous sclerosis: a population-based study. Dev Med Child Neurol, 1994. 36(1): p. 50-6.

13. de Vries, P.J., et al., A clinical update on tuberous sclerosis complex-associated neuropsychiatric disorders (TAND). Am J Med Genet C Semin Med Genet, 2018. 178(3): p. 309-320.

14. Bender, B.L. and E.J. Yunis, Central nervous system pathology of tuberous sclerosis in children. Ultrastruct Pathol, 1980. 1(3): p. 287-99. 
15. Nabbout, R., et al., Early diagnosis of subependymal giant cell astrocytoma in children with tuberous sclerosis. J Neurol Neurosurg Psychiatry, 1999. 66(3): p. 370-5.

16. Eker, R., Familial renal adenomas in Wistar rats; a preliminary report. Acta Pathol Microbiol Scand, 1954. 34(6): p. 554-62.

17. Waltereit, R., et al., Enhanced episodic-like memory and kindling epilepsy in a rat model of tuberous sclerosis. J Neurochem, 2006. 96(2): p. 407-13.

18. Rennebeck, G., et al., Loss of function of the tuberous sclerosis 2 tumor suppressor gene results in embryonic lethality characterized by disrupted neuroepithelial growth and development. Proc Natl Acad Sci U S A, 1998. 95(26): p. 15629-34.

19. Paxinos, G. and C. Watson, The Rat Brain In stereotaxic coordinates. 2007: Academic Press. 462.

20. Ovsepian, S.V., et al., Ambient Glutamate Promotes Paroxysmal Hyperactivity in Cortical Pyramidal Neurons at Amyloid Plaques via Presynaptic mGluR1 Receptors. Cereb Cortex, 2017. 27(10): p. 4733-4749.

21. O'Leary, V.B., et al., Innocuous full-length botulinum neurotoxin targets and promotes the expression of lentiviral vectors in central and autonomic neurons. Gene Ther, 2011. 18(7): p. 656-65.

22. Ovsepian, S.V., et al., Neurotrophin receptor $p 75$ mediates the uptake of the amyloid beta (Abeta) peptide, guiding it to lysosomes for degradation in basal forebrain cholinergic neurons. Brain Struct Funct, 2014. 219(5): p. 1527-41.

23. Bateup, H.S., et al., Excitatory/inhibitory synaptic imbalance leads to hippocampal hyperexcitability in mouse models of tuberous sclerosis. Neuron, 2013. 78(3): p. 510-22.

24. Nelson, S.B. and V. Valakh, Excitatory/Inhibitory Balance and Circuit Homeostasis in Autism Spectrum Disorders. Neuron, 2015. 87(4): p. 684-98.

25. Noroxe, D.S., H.S. Poulsen, and U. Lassen, Hallmarks of glioblastoma: a systematic review. ESMO Open, 2016. 1(6): p. e000144.

26. Bernal, A. and L. Arranz, Nestin-expressing progenitor cells: function, identity and therapeutic implications. Cell Mol Life Sci, 2018. 75(12): p. 2177-2195.

27. Lin, L., et al., Analysis of expression and prognostic significance of vimentin and the response to temozolomide in glioma patients. Tumour Biol, 2016. 37(11): p. 15333-15339.

28. Ito, D., et al., Enhanced expression of Iba1, ionized calciumbinding adapter molecule 1, after transient focal cerebral ischemia in rat brain. Stroke, 2001. 32(5): p. 1208-15.

29. Farnsworth, R.H., et al., Vascular remodeling in cancer. Oncogene, 2014. 33(27): p. 3496-505.

30. Takahashi, D.K., et al., Abnormal cortical cells and astrocytomas in the Eker rat model of tuberous sclerosis complex. Epilepsia, 2004. 45(12): p. 1525-30.

31. Wenzel, H.J., et al., Morphology of cerebral lesions in the Eker rat model of tuberous sclerosis. Acta Neuropathol, 2004. 108(2): p. 97 108.

32. Tschuluun, N., H.J. Wenzel, and P.A. Schwartzkroin, Irradiation exacerbates cortical cytopathology in the Eker rat model of tuberous sclerosis complex, but does not induce hyperexcitability. Epilepsy Res, 2007. 73(1): p. 53-64.

33. Lopes, M.B., et al., Immunohistochemical characterization of subependymal giant cell astrocytomas. Acta Neuropathol, 1996. 91(4): p. 368-75.
34. Hirose, T., et al., Tuber and subependymal giant cell astrocytoma associated with tuberous sclerosis: an immunohistochemical, ultrastructural, and immunoelectron and microscopic study. Acta Neuropathol, 1995. 90(4): p. 387-99.

35. Krinke, G.J., et al., Morphologic characterization of spontaneous nervous system tumors in mice and rats. Toxicol Pathol, 2000. 28(1): p. 178-92.

36. Nakamura, R., et al., Availability of a microglia and macrophage marker, iba-1, for differential diagnosis of spontaneous malignant reticuloses from astrocytomas in rats. J Toxicol Pathol, 2013. 26(1): p. 55-60.

37. Lendahl, U., L.B. Zimmerman, and R.D. McKay, CNS stem cells express a new class of intermediate filament protein. Cell, 1990. 60(4): p. 585-95.

38. Tohyama, T., et al., Nestin expression in embryonic human neuroepithelium and in human neuroepithelial tumor cells. Lab Invest, 1992. 66(3): p. 303-13.

39. Kitai, R., et al., Nestin expression in astrocytic tumors delineates tumor infiltration. Brain Tumor Pathol, 2010. 27(1): p. 17-21.

40. Mokry, J., et al., Nestin expression by newly formed human blood vessels. Stem Cells Dev, 2004. 13(6): p. 658-64.

41. Suzuki, S., et al., The neural stem/progenitor cell marker nestin is expressed in proliferative endothelial cells, but not in mature vasculature. J Histochem Cytochem, 2010. 58(8): p. 721-30.

42. Sugawara, K., et al., Nestin as a marker for proliferative endothelium in gliomas. Lab Invest, 2002. 82(3): p. 345-51.

43. Mendez-Ferrer, S., et al., Mesenchymal and haematopoietic stem cells form a unique bone marrow niche. Nature, 2010. 466(7308): p. 829-34.

44. Sorrells, S.F., et al., Human hippocampal neurogenesis drops sharply in children to undetectable levels in adults. Nature, 2018. 555(7696): p. 377-381.

45. Arai, H., et al., Nestin expression in brain tumors: its utility for pathological diagnosis and correlation with the prognosis of high-grade gliomas. Brain Tumor Pathol, 2012. 29(3): p. 160-7.

46. Mizuguchi, M., et al., Doublecortin immunoreactivity in giant cells of tuberous sclerosis and focal cortical dysplasia. Acta Neuropathol, 2002. 104(4): p. 418-24.

47. Boer, K., et al., Doublecortin-like (DCL) expression in focal cortical dysplasia and cortical tubers. Epilepsia, 2009. 50(12): p. 262937.

48. Kolenda-Roberts, H.M., et al., Immunohistochemical characterization of spontaneous and acrylonitrile-induced brain tumors in the rat. Toxicol Pathol, 2013. 41(1): p. 98-108.

49. Tremblay, R., S. Lee, and B. Rudy, GABAergic Interneurons in the Neocortex: From Cellular Properties to Circuits. Neuron, 2016. 91(2): p. 260-92.

50. De Palma, M., D. Biziato, and T.V. Petrova, Microenvironmental regulation of tumour angiogenesis. Nat Rev Cancer, 2017. 17(8): p. 457-474.

Publisher's Note Springer Nature remains neutral with regard to jurisdictional claims in published maps and institutional affiliations. 